\title{
Vulnerabilidades emergentes en procesos de relocalización forzada de personas
}

\author{
Emerging vulnerabilities in processes of forced relocation of people \\ Catalina Ramírez Vega ${ }^{1}$ \\ Carlos Arrieta Salas ${ }^{2}$
}

\begin{abstract}
RESUMEN
La noción de vulnerabilidad como algo intrínseco a una persona o a una población y que apunta a condiciones deficitarias, ha venido siendo desplazada por una concepción más dinámica y menos reduccionista, aquélla que en vez de hablar de vulnerabilidad como una condición atribuible al sujeto o al colectivo enfoca el tema más en términos de procesos de vulnerabilización. Desde esta perspectiva, en este artículo se analizan precisamente las vulnerabilidades emergentes que surgen en procesos de relocalización de personas que se vieron forzadas a dejar su comunidad como resultado de la declaración de inhabitabilidad provocada por los riegos a que estaban expuestas por las erupciones del volcán Chaitén en Chile. Con este propósito, se realizaron entrevistas a profundidad a personas de la Comuna de Chaitén relocalizadas en Futaleufú y mediante un análisis de contenido se identificaron cuatro dimensiones emergentes de vulnerabilidad: ruptura del tejido social, cambios en las condiciones de vida, cambios en las condiciones de salud y desinformación. A partir de estos hallazgos se analiza también el rol del Estado en la atención de esta emergencia, identificándose un "vacío psicosocial" significativo en el abordaje integral de la intervención con estas personas desplazadas de manera forzada de su territorio, por la prevalencia en este caso de un enfoque "emergencista" cuya atención se centra en apoyo material y económico dejándose de lado la atención de los aspectos psicosociales.
\end{abstract}

Palabras clave: Desplazamiento territorial forzado, Riesgo psicosocial, Desastres, Tejido social, Intervención social

\begin{abstract}
The notion of vulnerability as something intrinsic to a person or a population and pointing to deficit conditions, has been displaced by a more dynamic and less reductionist conception, which instead of speaking vulnerability as a condition attributable to the subject or the collective focuses the issue more in terms of vulnerability processes. From this perspective, this article analyzes precisely the emergent vulnerabilities that arise in relocation processes of people who were forced to leave their community as a result of the declaration of inhabitability caused by the risks to which they were exposed by the eruptions of the Chaiten volcano in Chile. With this purpose, in-depth interviews were carry out which people from the Chaitén community relocated in Futaleufú and through a content analysis, four emergent dimensions were identified: rupture of the social fabric, changes in living conditions, changes in the conditions of health and disinformation. Based in these findings, the role of the State in the care of this emergency is also analyzed, identifying a significant "psychosocial vacuum" in the comprehensive approach of the intervention with these people forcedly displaced from their territory, due to the prevalence in this case of an "emergencist" support, leaving aside the attention of psychosocial aspects.
\end{abstract}

Key Words: Forced territorial displacement, Psychosocial risk, Disasters, Social fabric, Social Intervention.

\footnotetext{
${ }^{1}$ Universidad de Costa Rica. Docente Escuela de psicología. Costa Rica. Correo electrónico:
} catalina.ramirez@ucr.ac.cr

${ }^{2}$ Universidad de Costa Rica. Doctor. Docente Escuela de psicología. Costa Rica. Correo electrónico: clas14@gmail.com Recepción: 15/11/2018 Aceptación: 26/3/2019 


\section{Introducción}

En el mes de mayo del 2008 hace erupción el Volcán Chaitén, ubicado en Chile en la región de Los Lagos, provincia de Palena, en la Comuna que da nombre al volcán. Como resultado de este evento natural las autoridades chilenas ordenan una evacuación total del pueblo, procedimiento que de acuerdo con Tapia (2012) no tenía precedentes en la historia chilena por la cantidad de personas que debían ser movilizadas, las formas de movilización empleadas y los medios de evacuación. Las personas que habitaban en Chaitén se relocalizaron en distintos lugares, unos más cercanos y otros mucho más alejados de su comunidad. Entre los primeros aparecen lugares como La Junta, Futaleufú, Palena (en promedio 150 kilómetros), Puerto Montt, Calbuco, Puerto Varas, La Unión, Osorno (en promedio 284 kilómetros) y otros sitios más alejados como Valdivia y Chiloé (en promedio 450 kilómetros).

La evacuación de Chaitén fue un proceso inestable, oscilante entre dos tendencias: el abandono del poblado y el retorno de los habitantes. Este proceso se dio a caballo entre el gobierno de la presidenta Bachelet y el gobierno del presidente Piñera. En el gobierno de la presidenta Bachelet, el proceso de evacuación se ocupó de la primera etapa de apoyo a la población chaitenina con acciones tales como el establecimiento de las familias en diferentes sectores, dotación de trabajo, arriendo de casas y otros beneficios. De seguido, y aún en medio de la emergencia, se abre la posibilidad de que los habitantes de Chaitén retornen a su comunidad (Contreras, 2008), sin embargo los estudios que se realizaron durante los periodos en que duró la emergencia y los posteriores a la evacuación, llevaron a que en el año 2009 se determinara que "el lugar original que tenía el poblado, no era apto para la vida humana, y se concluyó que la relocalización se haría en otro lugar costero ubicado a $10 \mathrm{~km}$ del lugar original" (Tapia, 2012, p.8). Los hallazgos encontrados en los estudios realizados indicaban que el territorio de Chaitén no era un lugar seguro para habitar, debido a las amenazas de nuevas erupciones volcánicas y a la posibilidad de que el asentamiento sufriera graves inundaciones. Esto lleva al gobierno a promover el desplazamiento y relocalización de la Comuna de Chaitén y a descartar la reconstrucción del pueblo (Jordana, 2009). 
Las ayudas económicas que brinda el Estado para atender esta situación de emergencia alcanzan los 25 mil millones de pesos, convirtiéndose en "el más importante aporte del Estado para [atender] una catástrofe natural” (Aguilera, 2009, p. 39). No obstante, a pesar de la "generosa disposición gubernamental" inicial, el proceso de relocalización de Chaitén se suspende bajo el argumento de que "el futuro de lo que pase con el repoblamiento será una decisión de otro mandato presidencial, porque los dineros para la construcción de un nuevo Chaitén no están incluidos en las partidas presupuestarias de 2009" (Aguilera, 2009, p.39). Sin embargo, al asumir el nuevo gobierno del presidente Piñera se dio un giro con respecto a la recomendación del diagnóstico original que sentenciaba la inhabitabilidad de Chaitén, afirmándose ahora que el pueblo sí podría ser reconstruido en el lugar original.

Este cambio de criterio dio origen a dos situaciones, a saber, por un lado el retorno de una parte de la población que nunca quiso abandonar el lugar a pesar de que las condiciones de riesgo se mantenían vigentes -aún hoy- y, por otro lado, la decisión de otra parte de la población de continuar viviendo en los lugares adonde fueron evacuados durante la emergencia y posterior a ésta. Futaleufú, localidad que se encuentra a 150 kilómetros de distancia por carretera de la Comuna de Chaitén, fue uno de los lugares de relocalización. En este lugar, se entregaron 118 viviendas a las familias de Chaitén que se trasladaron a vivir a esa comunidad desde el año 2008 (ver figura 1 y figura 2).

Las noticias divulgadas y los informes realizados en cuanto a lo sucedido en la Comuna de Chaitén, dan cuenta en general de la enorme inversión económica especialmente en infraestructura- que realizaron los gobiernos de Bachelet y de Piñera. Sin embargo, poco se ha dicho por los canales oficiales, acerca de las peripecias que han vivido las personas que debieron reconstruir su vida en otro sitio, lo que evidencia un vacío significativo en cuanto a procesos o acciones de apoyo psicosocial desde el Estado más allá del soporte material brindado. Este artículo pretende ocuparse de sistematizar un retazo del conjunto de historias tejidas en torno de este proceso de relocalización, centrando su atención allí donde han flaqueado las intervenciones estatales, a saber, en cuando a los procesos psicosociales propios de experiencias de migración forzada y las vulnerabilidades emergentes que se entretejen con las vulnerabilidades existentes antes de la situación de desastre. 

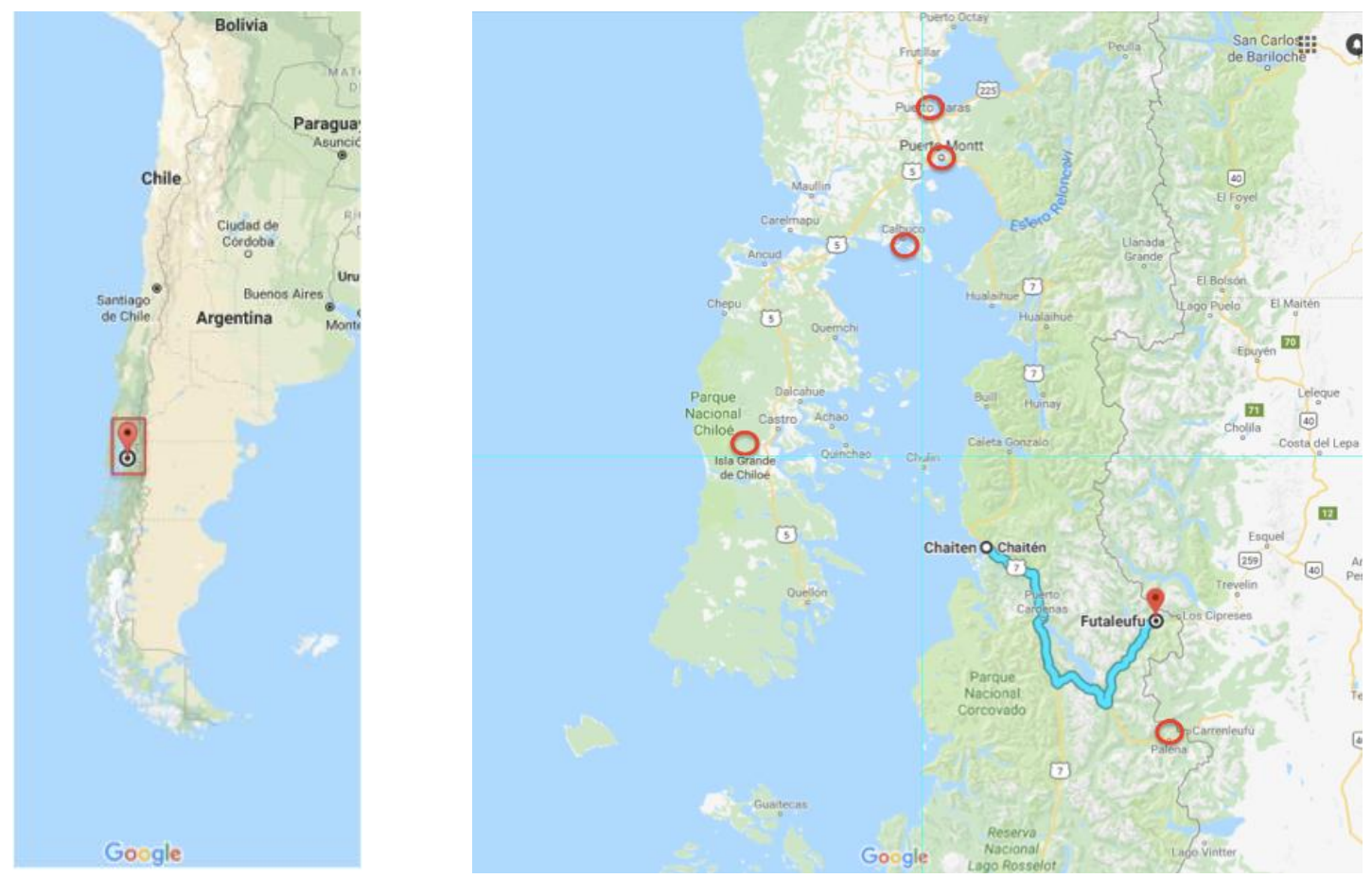

Figura 1 y Figura 2. Captura de imagen de Chile y de Chaitén (Fuente: Google Maps, 2018). En estas imágenes se observa a la izquierda el mapa de Chile con un recuadro que marca la zona de Chaitén y alrededores. A la derecha se observa una ampliación del recuadro en el que se pueden ver las comunidades de Chaitén y Futaleufú. Asimismo, encerrados en círculos rojos se muestran otros lugares donde se relocalizaron a personas de la Comuna de Chaitén. Otros lugares como Osorno, La Unión y La Junta no aparecen en el recorte de mapa por encontrarse a distancias mayores.

\section{Algunos elementos teóricos}

\section{Del riesgo a la construcción social del riesgo}

Cuando los desastres provocados por eventos naturales son concebidos por los Estados fundamentalmente como "eventos naturales", las acciones que se desarrollan para hacerles frente se centran generalmente en la recuperación de la infraestructura y se dejan de contemplar otros elementos de medular importancia como son los aspectos sociales y psicológicos. Es a partir del hallazgo de que la magnitud de los daños y las pérdidas derivadas de los desastres o eventos que afectan a los Estados tienen relación con las 
condiciones sociales, ambientales y económicas preexistentes, que, de acuerdo con Lavell (2001), se empiezan a generar nuevos espacios de reflexión en los cuales se considera y evalúa el impacto del desastre de forma más integral incorporando las condiciones socioambientales antecedentes. Es así como se avanza conceptualmente desde “... un punto de vista tradicional de auxilio y preparación frente a los desastres -en el cual las comunidades son consideradas como "víctimas" y "beneficiarias" de la asistencia brindada por expertos-" (Organización de las Naciones Unidas, 2001, p. D-1), a considerar las "condiciones preexistentes de vulnerabilidad de la sociedad" (Lavell, 2001, p.1) promoviendo la ejecución de acciones que tengan en cuenta estas condiciones preexistentes, tratando de minimizar hasta donde sea posible las consecuencias derivadas de un desastre. En este sentido, la prevención y mitigación de las consecuencias de los desastres resultará más efectiva en los ámbitos comunitarios, “... ya que es el ámbito comunitario donde los riesgos físicos, sociales y económicos se pueden evaluar y manejar de manera adecuada" (Organización de las naciones Unidas, 2001 p. D2)

$\mathrm{Al}$ entender los desastres desde esta perspectiva se vuelve una condición esencial antes de su ocurrencia "la reducción de la vulnerabilidad existente, y la promoción de esquemas de transformación de la sociedad que impiden la construcción en el futuro de nuevas condiciones de vulnerabilidad para la población" (Lavell, 2001, p.1). Teniendo esto en mente, tendrían los Estados y las instituciones que pensar formas alternativas de intervención y atención de las poblaciones que son afectadas por un desastre, considerando que "en muchas de las catástrofes naturales influyen de forma decisiva factores humanos y de toma de decisiones, además de la vulnerabilidad económica y política" (Berinstain, 2000, p.16)

Visto así el riesgo debe ser concebido de forma sistémica y como una construcción social, lo que lleva a trascender la concepción mecanicista que lo visualiza como el resultado de sumar amenaza+vulnerabilidad; de este modo se amplían las acciones para su reducción, dado que si se le concibe como una construcción "el proceso puede ser revertido, disminuido o recompuesto con medidas dirigidas a la reducción de la vulnerabilidad" (Perevochtchikova y Lezama, 2010, p.76). En congruencia con lo anterior, las estrategias han de pensarse más allá de "acciones reactivas a la emergencia"; debe contemplarse el ámbito político así como el desarrollo de "actividades de prevención, 
mitigación, preparación y atención de emergencias y recuperación post impacto" (Perevochtchikova y Lezama, 2010, p.76). En este sentido, es necesario insistir en que las acciones para la reducción del riesgo no deben limitarse a acciones de respuesta, dado que el nivel de riesgo dependerá de “...la producción y reproducción de las condiciones de vulnerabilidad que definen y determinan la magnitud de los efectos ante la presencia de una amenaza natural" (García, 2005, p. 23), poniendo en evidencia que los procesos de desastre parten de las vulnerabilidades previas en las que se encuentren las poblaciones.

\section{De la vulnerabilidad a los procesos de vulnerabilización}

La vulnerabilidad ha sido definida desde distintas disciplinas. A veces para explicarla llega a considerarse como una condición propia o interna que coloca a un sujeto o a una población en una predisposición de sufrir un daño. En otros casos, la vulnerabilidad se la hace depender del estado de la infraestructura o del contexto en el que se encuentre una población. De acuerdo con Lavell (2001), este concepto se desarrolla en ingeniería con el propósito de referirse básicamente a la resistencia de los elementos estructurales. A partir de esta metáfora, para comprender la vulnerabilidad, tanto en sus causas como en las consecuencias que se generan en contextos vulnerables, se crean modelos de vulnerabilidad multidisciplinar, en los cuales se articulan "los campos fîsiconatural, físico-espacial y socio-antropológico" (Chardon, 2008, p.125), y de los cuales derivan distintas clases de vulnerabilidad no excluyentes entre sí: físico-materiales, socialorganizacionales y motivacional actitudinales. (Anderson y Woodrow, 1989 en Chardon 2008).

La vulnerabilidad, de acuerdo con Chardon (2008), es un sistema complejo y dinámico que se expresa a través de factores que la producen; se trata de la "probabilidad de que un sujeto o elemento expuesto a una amenaza sufra daños y pérdidas [tanto] humanas como materiales en el momento del impacto del fenómeno, teniendo además dificultad en recuperarse de ello, a corto, mediano o largo plazo" (Chardon, 2008, p.126). Más adelante el mismo autor señala que un contexto vulnerable y por tanto vulnerabilizante "se gesta a lo largo de meses o años, puesto que se trata de un proceso generado por la 
sinergia entre factores que paulatinamente van creando esta situación de vulnerabilidad" (p.127).

A pesar de que teóricamente se empieza a establecer un cambio paradigmático que lleva al reconocimiento de la vulnerabilidad como "construcción", es decir como un proceso que no puede ni debe ser reducido a dinámicas endógenas de los sistemas, lo cierto es que si se mira el tema a partir de los modelos de desarrollo de los países (Lavell en Fernández, 2005) pareciera que "resulta más fácil negar la naturaleza estructural de la vulnerabilidad y el riesgo" (p.20), que implementar acciones que permitan su reducción, incidiendo directamente en la vulnerabilidad existente y evitando la producción de nuevas condiciones de vulnerabilidad. Sin embargo, como estas acciones requieren "mecanismos de compensación social que exceden por mucho las posibilidades y capacidades financieras o la voluntad política de los gobiernos -aún de los más avanzados en concepción social y humanitaria-." (Lavell en Fernández, 2005, p. 23), lo más frecuente es que se sigan enfrentando los riesgos y amenazas de la manera tradicional.

Para Fernández (2012) la vulnerabilidad no es "ni casualidad, ni destino, sino que, para que un grupo social estuviera ubicado en ese camino a la exclusión fueron necesarias expresas políticas de Estado que, a través de los años y de diferentes gobiernos, produjeron activamente dicha vulnerabilidad" (p.66). Para muestra de lo anterior lo ocurrido en la tragedia provocada por el Huracán Katrina en la que, tal y como lo señala Bauman (2011), lo que "sucedió en esos momentos vino determinado por todo lo ocurrido con anterioridad al desastre en sí, es decir, por los seres humanos y las decisiones que tomaron"3 (p.107)

La vulnerabilidad debería entenderse como "una construcción activa, un producto, un proceso y no meramente un hecho o dato, que una larga y variada serie de decisiones económicas, políticas y sociales habían finalmente logrado configurarla” (Fernández, 2012, p.66)

\footnotetext{
${ }^{3}$ Bauman desarrolla más en extenso este tema indicando que si bien durante el Huracán Katrina el sistema de alerta temprana funcionó y que las personas estaban informadas con respecto al huracán, "no todos pudieron actuar conforme a lo que ya sabían ni hacer un buen uso del tiempo del que disponían para la huida. Algunos no pocos- fueron incapaces de reunir suficiente dinero para comprarse billetes de avión... [El Huracán] podría haber atacado a los culpables y a los inocentes, a los ricos y a los pobres con la misma ecuanimidad, pero una catástrofe reconocidamente natural como aquella, no fue percibida como igualmente natural por todas sus víctimas." (Bauman, 2011, p.104).
} 
De acuerdo con Bauman (2011), los terremotos, las inundaciones, los huracanes y eventos similares son naturales ya que no son en sentido estricto eventos provocados directamente por la acción humana, pero las consecuencias que se desprenden de estas situaciones sí se constituyen en un producto humano. Es decir, que las vulnerabilidades que emergen en las poblaciones afectadas por un evento natural van a estar relacionadas con las acciones desplegadas o no por los Estados y las acciones que desarrollen las personas. Si estas vulnerabilidades emergentes no se incorporan activamente en los modelos de atención, se estarán creando condiciones de vulnerabilización que harán más severas las consecuencias del desastre.

En este sentido, y siguiendo a Rosales y Salazar (2010), los procesos de reconstrucción y relocalización posteriores a un desastre provocado por un evento natural no deben reproducir vulnerabilidades existentes y menos generar nuevas vulnerabilidades. Esto implica que estos procesos no deben centrarse únicamente en la reposición de las estructuras físicas, de los caminos, de las viviendas, sino también incorporar acciones con respecto a aspectos sociales y psicológicos trascendentales como son el arraigo, las costumbres, la reconstrucción del tejido social, el acceso a fuentes de trabajo y el derecho a la propiedad, es decir, se deben generar estos procesos incorporando un enfoque psicosocial que tradicionalmente ha estado excluido.

\section{El desplazamiento por desastres}

Cualquier desplazamiento forzado de personas o poblaciones resulta mínimamente difícil y complejo y lamentablemente en muchos casos muy doloroso y traumático. En lo que respecta a desastres por eventos naturales ha habido un enorme vacío en cuanto a normativa local e internacional para regular el abordaje de situaciones que implican la relocalización de personas. Este vacío se ha visto paliado con la redacción de los Principios Rectores sobre el Desplazamiento Interno, elaborado por ACNUR (1998) para aplicar en casos de desplazamientos forzosos por motivos relacionados principalmente con violencia política y social. Si bien es cierto no tiene carácter vinculante al menos ha ido adquiriendo reconocimiento y no sin difícultades ha incluido el desplazamiento por desastres asociados a eventos naturales. De acuerdo con Cohen (2009) en su momento "apenas había consenso 
sobre sí se debía incluir [en los Principios] los derechos de los que habían quedado desarraigados debido a los desastres". Tal y como lo ve este autor, desde los aparatos institucionales no se considera a estas personas como desplazadas, sino que se les reconoce aplicando otras categorías tales como "desarraigadas..., evacuadas, y por último, víctimas del desastre, porque en su opinión, los desplazados internos son personas desplazadas debido a conflictos armados en otros lugares" (Cohen, 2009).

En la revisión de antecedentes realizada para desarrollar este artículo, se identificó un vacío significativo en cuanto al estudio de las necesidades de estas poblaciones post desastre, lo cual implica una limitación muy importante en la generación de mecanismos institucionales adecuados para afrontar integralmente las necesidades que deben ser atendidas. Lo que las investigaciones informan es que la atención se ha concentrado en entregar viviendas, cuando en definitiva el desplazamiento implica muchas más pérdidas que las que pasan por el ámbito material-estructural. Otro elemento que es necesario considerar es que "este tipo de desplazamiento violento y de corta duración puede convertirse en migración permanente o temporal cuando el fenómeno cambia radicalmente las condiciones previas del ambiente natural” (Adamo, 2001; p.153). En estos casos, continúa señalando Adamo, el movimiento que deben realizar las poblaciones afectadas por un desastre no es solo involuntario, sino que en ocasiones se torna violento, repentino y en algunos casos francamente caótico. Ello implica, como indica Rojas (2010), que las personas deben enfrentarse al desarraigo, a la pérdida de los seres queridos, el abandono de la tierra y en ocasiones, hasta la salida de su región, lo que produce en las personas, según han encontrado Palacio y Madariaga (2006), “diversos sentimientos de impotencia, tristeza, ansiedad y depresión" (p.91).

A lo anterior se suma que las personas experimentan dificultades de integración en las comunidades donde se instalan, lo cual de acuerdo con Palacio y Madariaga (2006, p.89) se desprende de la forma en que estas personas llegan a las nuevas comunidades, donde en definitiva "en la interacción de ambos grupos (desplazados o los que llegan y receptores o asentados) se construye el contexto en el que se dará la adaptación temporal o definitiva al nuevo ambiente de vida para todos en la comunidad". Estudios clásicos (Elias y Stockton, 2000; Schütz, 2012; Simmel, 2012) advierten claramente de las dificultades que 
emergen cuando outsiders ${ }^{4}$ se instalan en comunidades establecidas. Estos estudios indican que la reacción de las personas que se auto perciben como originarias de un poblado tienden a rechazar a las personas recién llegadas por considerarlas amenazantes, peligrosas, fuente de malas prácticas, y de un status moral inferior, todo esto con independencia de las características que efectivamente comportan dichas personas. La figura 2 ilustra de manera magnífica está dinámica.

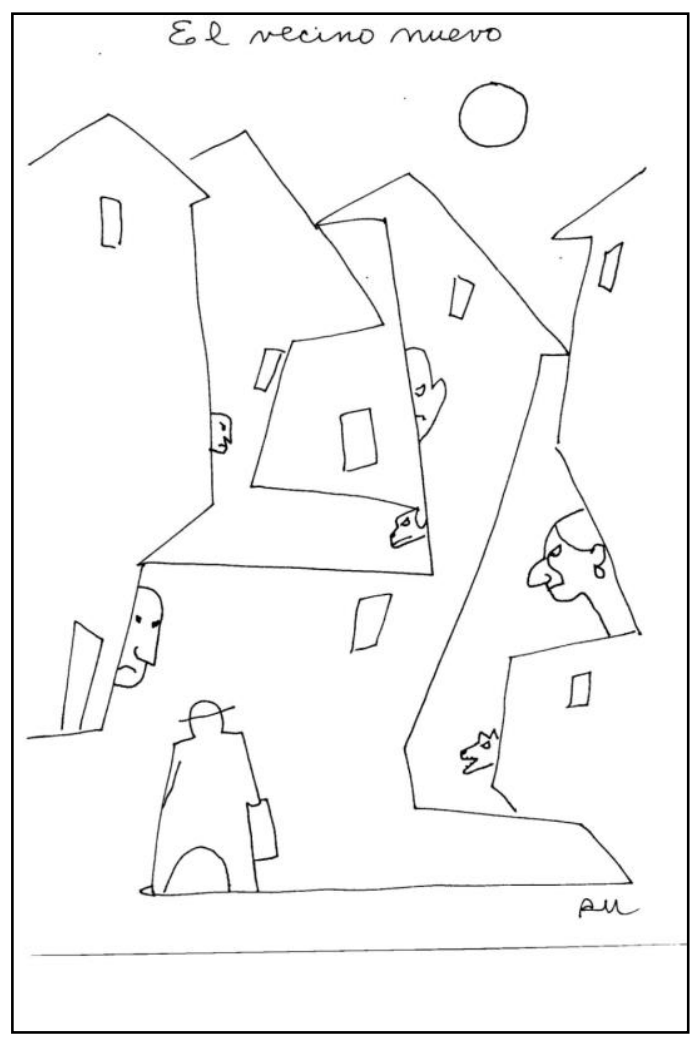

Figura 3. El Vecino Nuevo. Dibujo que expresa de forma gráfica muchas de las actitudes y comportamientos que muestran los viejos vecinos de la comunidad ante la llegada de un forastero. Reproducido con autorización del artista y psicólogo Ricardo Ulloa Garay.

\footnotetext{
${ }^{4}$ Este es el término empleado por Elias y Stockton para referirse a las personas recién llegadas a la nueva comunidad. En las traducciones al castellano este término ha sido traducido como "marginales". Evidentemente está atribución resulta de una doble percepción, a saber, la del viejo vecino que ve como extraño al recién llegado y la del recién llegado que se ve doblemente como extraño a partir de sus propias sensaciones sumadas a las percepciones de los viejos vecinos. El término que utiliza Schütz (2012) para referirse a recién llegados es el de "forasteros", mientras que Simmel (2012) los denomina extranjeros. En todas las connotaciones el recién llegado, que llega con la expectativa de establecerse, es visto como un cuerpo extraño que genera, unas veces más otras menos, interacciones hostiles nacidas de atribuciones negativas.
} 
Si entendemos la comunidad -aquella de la cual han de ser desplazadas las personas- como una organización que tiene componentes territoriales y afectivos (Sánchez, 2007) que nutren y sostienen los procesos de arraigo y pertenencia, habremos de suponer de inmediato que las personas desplazadas de manera forzada se enfrentan a la situación de tener que desprenderse del territorio y en alguna medida - unas más, otras menos- de la red de vínculos afectivos y socioemocionales, ya que esta lejanía geográfica paulatinamente va colonizando los vínculos emocionales fuertemente asociados a lo espacial y a lo temporal. En el mejor de los casos, procesos de hibridación (García Canclini, 2001) ${ }^{5}$, habrán de brindar nuevos soportes emocionales que facilitarán nuevos arraigos, sin embargo, esto no está garantizado de antemano ya que hay barreras que los dificultan (p.ej. el rechazo de los viejos vecinos, la idealización de la vieja comunidad, los duelos no resueltos, etc.). De acuerdo con Duque (2006) una comunidad que requiere ser reasentada sufre "nuevos impactos y consecuencias, que afectan su propio equilibrio y normal desarrollo, situaciones que el Estado tiene el deber de atender ágilmente" (p.146). En consecuencia, uno de los principales cambios a los que se enfrentan las poblaciones que son desplazadas es el establecimiento de nuevas relaciones sociales y nuevas formas de cooperación, dado que deben en un primer momento insertarse en otra comunidad, y posteriormente, si se da un proceso de reubicación, reconstruir el tejido social con las antiguas personas de su comunidad.

En cuanto a las vulnerabilidades generadas por procesos de desplazamiento forzado, diversos autores han encontrado que el desarraigo afecta la relación de la persona consigo misma, así como la construcción de vínculos con otras personas y la relación que establecen con su entorno (Arrieta, 2017, Berroeta, Ramoneda, Rodríguez, Di Masso y Vidal, 2015; Grinberg y Grinberg, 1984, 1998;). En una revisión hecha por Arrieta (2017), el autor encontró que “...en el plano individual las consecuencias psíquicas (emocionales y cognitivas) que se experimentan como resultado del desarraigo y la falta de apoyo social constituyen una fuente de sufrimiento y malestar para las personas y, en el plano colectivo, ambas condiciones generan un debilitamiento de los lazos sociales y de las relaciones de

\footnotetext{
${ }^{5}$ A decir de García Canclini: “...entiendo por hibridación procesos socioculturales en los que estructuras o prácticas discretas, que existían en forma separada, se combinan para generar nuevas estructuras, objetos y prácticas" (p.14).
} 
interdependencia. (p. 129). Por su parte Uribe y Salinas (2011) encuentran que: "Estas condiciones materiales y simbólicas de vida generan malestar y cambios en la subjetividad y una retahíla de consecuencias negativas como la pérdida de la identidad social, fragmentación de los lazos sociales, vulnerabilidad, aislamiento, individualismo entre otras además de la construcción de sujetos fragmentados e inseguros, es decir, genera precarización psíquica. (Uribe y Salinas, 2011, p.269).

En este estudio, cuyo enfoque radica en identificar y sistematizar vulnerabilidades emergentes en el caso de Chaitén, veremos cuáles son las vulnerabilidades emergentes específicas en esta Comuna y qué relación guardan con las estrategias y procedimientos empleados para atender la crisis derivada de este desastre provocado por la erupción del volcán que da nombre a la Comuna.

\section{Metodología}

Para llevar a cabo la investigación que da origen a este artículo se utilizó un enfoque cualitativo en el cual la producción de conocimiento es concebida como una construcción compartida, a partir de la interrelación investigador-investigado. Wiesenfeld (2000) sostiene que la investigación cualitativa reivindica la relación que se da entre la persona que investiga y la persona que brinda la información, dado que tanto las experiencias de vida como los significados que las personas le atribuyen a éstas son trasmitidas en el marco de una relación de horizontalidad, atravesada por el respeto mutuo, por el diálogo reflexivo. En este sentido, Canales (2006) señala que dentro de este enfoque, el investigador "se mueve en el orden de los significados y sus reglas de significación: los códigos y documentos, o significaciones" (p.19), teniendo como objetivo lograr la "reproducción de la comunidad o colectivo de hablantes de una lengua común para su análisis y comprensión” (p.19).

La investigación se llevó a cabo con personas que fueron desplazadas de sus comunidades por la situación de desastre, y que se vieron forzadas a reiniciar su vida en otro lugar, siendo beneficiadas con viviendas de ayuda social entregadas por parte del Estado. El muestreo utilizado fue intencionado. En total se realizaron 9 entrevistas a personas originarias de Chaitén que actualmente viven en la población Villa el 
Bosque en Futaleufú. Para participar en la investigación las personas a entrevistar tenían que cumplir con tres criterios de inclusión: a) ser hombres o mujeres mayores de 18 años, b) que fuesen residentes de Chaitén en el momento en que se dieron las erupciones del volcán y c) ser beneficiarios de una casa proveída por parte del Estado, o bien, haber recibido subsidios que les permitiera acceder a una vivienda.

Para establecer contacto con los y las participantes se realizaron varias visitas a la comunidad con el fin de identificar informantes clave, se les explicó en qué consistía la investigación, se conoció el contexto en el que viven y se acordó con cada persona que en próximos encuentros se realizarían las entrevistas. En el primer contacto directo con las personas entrevistadas se les explicó los detalles objetivos y alcances de la investigación, se describieron los componentes de la entrevista y se indagó sobre el estado emocional de las personas Este último procedimiento reviste mucha importancia dado que en la entrevista se exploran diversos momentos antes, durante y después de la emergencia, los cuales podrían resultar muy movilizadores y no se quería generar procesos emocionales que pudieran desbordar a las personas o, peor aún, que resultaran en una re-victimización de ellas. Una vez que se determinó si la persona podía y estaba anuente a conversar acerca de su experiencia, se procedió a concertar una reunión en el horario que le fuera conveniente para realizar la entrevista ${ }^{6}$.

Para definir el número de personas a entrevistar, así como la cantidad de entrevistas a realizar a una misma persona se empleó el criterio de saturación que, siguiendo a Álvarez-Gayou (2003), se define como el momento en que durante el proceso de producción de la información, ésta empieza a ser redundante -similar o repetitiva- dejando de aportar nuevos elementos o insumos. Con respecto de esto Canales (2006) señala que "la representación cualitativa opera por el principio de redundancia o saturación, entendiendo por ello el agotamiento de información o efectos de sentido no conocidos previamente" (p.23). Es así como a partir de la octava persona entrevistada dejaron de

\footnotetext{
${ }^{6}$ Aun cuando se realizaron estas primeras conversaciones con las personas con el fin de determinar su disposición y fortaleza emocional para hacer la entrevista, en casi todos los casos hubo que brindar en algún momento apoyo psicológico (intervención en crisis) cuando se abordaron temas difíciles que movilizaban grandes montos de afecto o bien momentos en donde la persona se sentía frágil al tratar ciertos temas. La experiencia de la coautora de este artículo en intervención en crisis resultó vital para dar acompañamiento psicológico a estas personas orientando el proceso de modo que la misma entrevista resultara en cierta medida terapéutica para la persona.
} 
aparecer nuevos elementos narrativos que resultaran significativos, produciéndose una redundancia que indica que "el objeto se ha agotado en sus descriptores" (Canales, 2006, p.24). No obstante, para asegurarse de que fuera así se entrevistó a una novena persona con la que se confirmó que efectivamente los procesos relatados por las personas giraban recurrentemente en torno de un conjunto de dimensiones que se describirán más adelante.

La tabla 1 presenta información descriptiva las personas participantes en la investigación, todas aparecen identificadas con un código (E1, E2, así sucesivamente) con el propósito de guardar confidencialidad y proteger el nombre de las personas.

Tabla 1.

Participantes del estudio

\begin{tabular}{ccc}
\hline Identificació & Edad & Sexo \\
\hline E1 & 25 & Femenino \\
E2 & 28 & Masculino \\
E3 & 41 & Femenino \\
E4 & 43 & Femenino \\
E5 & 41 & Femenino \\
E6 & 38 & Femenino \\
E7 & 50 & Femenino \\
E8 & 42 & Femenino \\
E9 & 56 & Femenino \\
\hline
\end{tabular}

Con respecto de la población participante es importante hacer un par de aclaraciones. En primer lugar, señalar que aunque la intención fue siempre incluir hombres y mujeres, la muestra quedó prácticamente compuesta solo por mujeres debido a que del total de personas a las que se invitó a participar las que consintieron ser entrevistadas fueron ellas. En segundo lugar, señalar que una de las entrevistas fue realizada en familia con la participación de la pareja y los hijos, pero se ha clasificado como participante mujer debido a que la "voz cantante" durante toda la entrevista la llevó siempre la mujer. En tercer lugar, importante tener presente que el que la muestra haya estado prácticamente compuesta por 
mujeres puede introducir un sesgo en la información producida y forzar redundancias mediadas por la condición de género. Si bien la información aportada por el hombre entrevistado coincidió en mucho con lo dicho por las mujeres, el hecho de ser solamente un varón entrevistado impide generalizar de alguna manera sus apreciaciones e impide también apresurar conclusiones con respecto a similitudes y diferencias según género.

\section{Técnicas de producción de información}

Como se dijo líneas atrás, la técnica de producción de información utilizada fue la entrevista. De los diferentes tipos de entrevista se seleccionó la entrevista semiestructurada, en la medida en que ésta "hace referencia a un cuestionario abierto, es decir a la flexibilidad que genera la interacción para construir el relato, siendo la emergencia de preguntas parte de la situación conversacional” (Labrín, 2010, p.43), lo que permite ir reconstruyendo la experiencia de la persona e ir identificando no solo los eventos emergentes sino también tomarle el tempo a la entrevista, identificando los momentos cuando se puede profundizar aún más en los procesos apreciativos. Igualmente, esta técnica se consideró adecuada para alcanzar los objetivos propuestos (tanto la caracterización de las vulnerabilidades como la identificación de las acciones que realizaron las personas ante éstas), dado que de acuerdo con Bordas (2000) permite analizar "el sentido que los actores le dan a sus prácticas y a los acontecimientos que confrontan: sus interpretaciones de situaciones conflictivas o no, las lecturas de sus propias experiencias" (p.185). Las dimensiones exploradas en la entrevista fueron de carácter temporal (el antes, el durante y el después de la relocalización) poniendo mayor atención en las vulnerabilidades y riesgos percibidos por las personas entrevistadas en cada uno de esos tres momentos. En virtud de esto, la guía de entrevista invitaba a las personas entrevistadas a transitar por los momentos previos al desastre, por el momento de la emergencia, de la evacuación, del desplazamiento y, finalmente, por las condiciones actuales. La entrevista transcurría como una conversación donde preguntas generadoras y comentarios iban abriendo camino por los espinosos temas que se requería explorar.

Por último, es importante indicar que cada una de las entrevistas fue realizada por un equipo de dos profesionales en psicología con experiencia en investigación de 
campo en comunidad y en procesos de relocalización de personas originados por desastres provocados por eventos naturales. Uno de los miembros se encargaba de conducir la entrevista mientras el otro miembro verificaba que se fueran abarcando todos los temas contenidos en la guía de entrevista y registrando los procesos emergentes tanto cognitivos como emocionales.

\section{Análisis de la información producida}

La técnica elegida para analizar la información producida fue el análisis contenido. Según Aigneren (1999), lo que esta técnica propone es investigar el contenido de las comunicaciones por medio de la clasificación en categorías de los elementos o contenidos manifiestos en comunicaciones o mensajes. Por su naturaleza esta técnica posibilita analizar "con detalle y profundidad el contenido de cualquier comunicación" (Porta y Silva, s.f., p.8). Otra de las razones por las se optó esta técnica es porque, como indica Aigneren (1999), permite realizar análisis tanto descriptivos como inferenciales, "por lo que el análisis no está limitado al contenido manifiesto de los mensajes, sino que puede extenderse a su contenido latente". (p.6)

Para el análisis del material producido se procedió en un primer momento a hacer una transcripción literal de las grabaciones de las 9 entrevistas. Una vez transcritas se procedió a verificar la calidad de las transcripciones contrastando el audio con la versión escrita. Una vez hecho lo anterior se procedió a:

1. Realizar una lectura a profundidad de las entrevistas. Para ello se leyeron varias veces con el propósito de tener una idea lo más completa posible de la información producida e ir identificando elementos comunes en las entrevistas.

2. Codificar la información procediendo a señalar frases, párrafos o temas que se relacionaran directamente con el objetivo de esta investigación. Posteriormente se establecieron tópicos, y se hizo lo que recomiendan González y Cano (2010), es decir generar "un listado de los mismos explicando la significación que el investigador atribuye a cada uno de ellos". (p. 6). Acto seguido se identificaron los tópicos que tenían un mismo significado con el propósito de generar reagrupaciones o clústeres a partir de los cuales establecer las categorías. Posteriormente en sesiones de trabajo con un grupo 
de profesionales seleccionados por tener amplia experiencia en la intervención en desastres y en psicología comunitaria se sometieron validación las reagrupaciones realizadas.

Una vez que se realizaron los pasos anteriormente descritos, se procedió a efectuar la exposición de los datos, identificando nuevas interrelaciones que anteriormente no fueron visibles. Asimismo, se elaboraron memos en los procesos, tanto de fragmentación de la información, de la codificación y construcción de categorías, ya que de acuerdo con González y Cano (2010), esto se convierte en un elemento para generar posteriormente las reflexiones analíticas. En este sentido, las autoras señalan que estos memos se convierten en "un procedimiento de recogida de insights (reflexiones críticas sobre el proceso de investigación, e inferencias analíticas a partir de los datos)" (González y Cano, 2010, p.8), que son muy útiles en el proceso de análisis. Una vez realizado todo el proceso descrito se procedió a generar identificar las vulnerabilidades emergentes asociadas a cada una de las categorías que fueron apareciendo en los análisis.

\section{Resultados}

El análisis de contenido realizado permitió identificar cuatro categorías básicas referidas de forma directa a vulnerabilidades emergentes, a saber: 1) Ruptura del tejido social, 2) Cambios en las condiciones de vida, 3) Cambios en las condiciones de salud y 4) Desinformación. En la tabla 2 aparecen descritas estas cuatro categorías así como los tópicos más específicos que les dan contenido. 
Tabla 2

Vulnerabilidades emergentes y tópicos identificados en la población

Categorías

1. Ruptura del tejido social

2. Cambios condiciones de vida

3. Cambios condiciones salud

4. Desinformación

\section{Tópicos}

1.1. Desintegración familiar

1.2. Ausencia-pérdida de redes de apoyo

1.3. Rechazo de las comunidades receptoras.

1.4. No tener sentido de pertenencia, desarraigo.

2.1. Falta de empleo.

2.2. Arriendos

3.1. Aparición de enfermedades.

3.2. Dificultades en el estado de ánimo.

4.1. No conocer lo que está sucediendo.

A continuación, se describe más en extenso cada una de estas categorías que se acompañan de fragmentos de entrevista que en este caso deberán entenderse como "retazos" representativos del sentir de las personas entrevistadas

\section{Categoría 1. Ruptura del tejido social.}

Esta categoría corresponde a las descripciones que hacen las personas entrevistadas con respecto de las rupturas que sufrieron a partir de la evacuación de Chaitén y la desarticulación de las tradicionales redes de apoyo por la relocalización de sus familiares en distintas zonas geográficas muy distantes entre sí. Las frecuentes referencias a experiencias de soledad, de no contar con nadie cercano en quien apoyarse aparecen en su cotidianidad después de la salida de Chaitén. Asimismo, dentro de esta categoría se enmarca el rechazo de las comunidades donde llegan a instalarse, con lo cual la sensación de estar solos y solas se incrementa. 


\subsection{Desintegración familiar}

Las personas entrevistadas mencionan que a partir de la evacuación sus familias quedan dispersas en diferentes partes del sur de Chile y que la reunificación ha sido un proceso complicado. Asimismo, aún cuando hay familias que lograron reagruparse casi totalmente, hubo momentos entre la evacuación y la entrega de sus respectivas viviendas en los que experimentaron la desintegración familiar y la desolación concomitante.

“... la familia: mi mamá en Puerto Montt, mis tíos en Puerto Montt, otro tío en Palena .. yo estaba solo acá, no había nadie más, eso fue lo más difícil.” (E1)

“... al principio fue difícil, estuve como separada dos meses de mi hijo, el chiquitito; nosotros al principio nos fuimos, con la misma jefa que estoy trabajando ahora, nos fuimos a La Junta. Claro lo que más me afectó al principio fueron esos dos meses que no estuve con él, que no estaba con mi hijo y yo era una madre de esas aprehensivas a morir, que no dejaba que mi hijo saliera." (E5)

“... Y fue eso, fue difícil, hasta los días de hoy no hemos logrado juntarnos todos de nuevo. Entonces fue fuerte, fue como que nos hubieran arrancado algo y se extraña: se extraña a la familia, se extraña a mi papá, en realidad ya no es lo mismo.” (E6)

\subsection{Ausencia de redes de apoyo.}

Las personas entrevistadas mencionan que a partir de la desintegración familiar y al empezar a vivir en un lugar distinto, no cuentan con apoyo para solucionar las dificultades que se les presentan, las cuales pueden ser de cuidado de sus hijos e hijas, o bien, ante los problemas que se presentan en la vida cotidiana.

“... si, el dejar a la Carla sola, porque había días en que yo tenía que, porque salía más tarde... entonces era como si mi pareja, mi pololo no me ayudaba, era como el único que me tenía que ayudar jajaja, en ese momento porque era la persona a la que tú le tienes confianza o que te ayudaba, o que él sabía en las circunstancias en que estábamos nosotras." (E2). 
“... hay cosas que cuando uno siente que la familia está al lado quizás no duelen tanto. O sea, igual duelen, pero tú sientes que ellos te están apoyando y que tú no vas a estar sola. O sea, te puede faltar cualquier cosa pero van a estar ellos. Yo creo eso... pero acá, si yo dejo de trabajar y me faltara de comer: ¿a quién voy a pedirle? ¿a dónde voy y le digo: sabe qué hoy no tengo qué cocinar?. No puedo". (E5)

\subsection{Rechazo de las comunidades receptoras.}

En las entrevistas, las personas comentan de las actitudes de rechazo que han experimentado por parte de los vecinos de Futaleufú, así como el enojo que experimentan cuando se atribuye a su llegada robos y conflictos en la comunidad sin visibilizar los aspectos positivos o el crecimiento que ha tenido Futaleufú a partir de la llegada de las personas relocalizadas allí. Esto incidió e incide en el proceso de adaptación de las personas.

“... yo creo que fue el hecho de llegar acá, que había mucha gente que no conocíamos y la gente no fue muy amable al principio, entonces como que uno veía y muchas veces comentaba cosas la gente de que, que por qué nos habíamos venido para acá sino hay casa, y que esto y que lo otro; entonces como que yo pienso que eso fue complicado". (E3)

"Y después otra cosa que los chaiteninos fuimos mal vistos también, acá en Futa. No por toda la gente, pero sí había gente: “ah, esos”. Se perdía algo acá en Futa y le echaban la culpa a la gente de Chaitén, y sabe que finalmente pillaban a la gente y eran los mismos de Futa que robaban. Entonces todas esas cosas como que la gente sintió mucho rechazo en eso: "y llegaron los chaiteninos, los chaiteninos aquí", éramos como la escoria de...". (E7)

“...como que se empezó a formar una rivalidad porque era, era como que nosotros veníamos a gastarle lo que era de ellos”. (E4)

“... yo digo, hay mucha gente que ha sido muy buena, hay que gente nos ha ayudado mucho, que apoyó mucho cuando recién llegamos pero hay gente que igual nos ha hecho sentir como que no somos de acá, como que el pueblo no nos 
pertenece... como que no somos... como que si roba algo, no es la gente de acá, es la gente que vino: somos nosotros. Como que si hay basura en la calle, no es la gente de acá la que bota basura, es la gente que llegó”. (E6)

“... entonces llegar acá y tener que pagar un arriendo tan costoso y la misma gente igual no, como que de primera a nosotros no, como que nos miraban feo así como que les habíamos venido a invadir su pueblo por así decirlo; entonces como que eso igual a nosotros también nos costó. Porque había, si bien había gente que igual nos recibía bien, pero había gente igual que de repente era agreste cuando usted iba a comprar al negocio y cosas así. Y uno lo notaba así en el ambiente, porque uno veía como a la gente de acá lo trataban bien, y a uno así como medio áspero, uno se da cuenta cuando a uno lo reciben bien y cuando no". (E3)

\subsection{No tener sentido de pertenencia, no querer estar aquí. Desarraigo.}

En el discurso de las personas entrevistadas aparece que habitar en Futaleufú no es fruto de su deseo, que dejar Chaitén fue por obligación y que Futaleufú, fue elegido por tener características similares con Chaitén.

“... o ambas cosas, porque te cambió la vida, o sea, se dio un giro total, total, no es a un lugar donde tú te quieras ir a vivir con alguien, a estar prácticamente porque no hay otro lugar. O sea, la Nexi vivía acá, pero uno no... a lo mejor si me hubiera juntado con la Nexi le hubiera dicho "vamos a vivir a Chaitén", por ejemplo. O venir, y ya por último nos quedamos acá, pero ya venir con la mente dispuesta a eso, aquí fue por fuerza mayor". (E1)

“...claro, eso fue, es que ese es el problema... si nosotros no salimos por elección, tuvimos que salir por obligación. Pero sí yo digo que eso lo superaremos”. (E7)

\section{Categoría 2. Cambios en las condiciones de vida.}

Esta categoría contempla las dos situaciones que las y los entrevistados mencionan constantemente en sus conversaciones con respecto de los cambios que debieron enfrentar en su calidad de vida, lo cual se dio a partir de la evacuación de 
Chaitén. Estas dos situaciones se centran específicamente en la necesidad de arrendar una casa y la falta de empleo.

\subsection{Falta de empleo.}

Las personas entrevistadas señalan que una de las mayores dificultades que deben enfrentar es la pérdida de empleo a partir de la evacuación de Chaitén y el desplazamiento a diferentes lugares. Esto, a pesar de las ayudas que reciben del Estado, se convierte en una fuente de estrés para las familias, dado que su calidad de vida disminuye ante las nuevas circunstancias. Es importante señalar que esta dificultad es experimentada, principalmente, en la evacuación y en los momentos posteriores, y que en la situación actual de las familias esto ya no se percibe como una dificultad mayor.

“... aquí había estado trabajando, entonces yo iba a encontrar un jefe en Puerto Montt, yo manejaba un camión, y el jefe me dijo: “no, ándate no más, si estás allá trabajo vas a tener". Me vine con esa esperanza... nada no me dieron ni bola...” (E1)

“... estuvimos como dos meses viviendo en La Junta mientras, porque estaba cerrado Futaleufú, y como nosotros trabajábamos con eso stile, porque en ese tiempo teníamos una tienda en Chaitén de ropa y claro lavandería, y eso, pero ya no, entonces dónde vamos a trabajar po', porque teníamos que seguir trabajando”. (E5)

\subsection{Arrendar}

Las dificultades nacidas de la necesidad de arrendar constituyen una preocupación y fuente de agobio constante para estas personas relocalizadas porque pasaban de ser propietarios de su espacio a inquilinos en un espacio rentado. Al impacto emocional que supone este cambio de condición se le agrega el hecho de tener que arrendar casas en muy mal estado, las cuales en cualquier momento tenían que desocupar porque se las pedían para arrendárselas a otras personas, lo que generaba inseguridad. De acuerdo con las personas entrevistadas, al darse a conocer el monto del subsidio del gobierno para el alquiler se dieron manejos difíciles debido a la especulación por parte de los dueños de las viviendas. 
“... entonces ya tuvimos que empezar a tomar decisiones así como a arrendar una casa para varios meses. Mi mamá tenía su negocio, yo solo trabajaba. Ya, empezar un local para el negocio, y bueno acostumbrarse a ese... era distinto porque el negocio antes lo teníamos en la casa, estabas ahí mismo con tu hija y todo, ahora el negocio quedaba lejos". (E2)

“... entonces era como estar súper penca de andar de, "no, que vamos pa’allá, que vamos pa'acá" y que esto, cambiarnos de casa, de que las casas son caras, después ya empezaron a subir los arriendos, y todo era un caos porque ya como empezaron ya, la gente tenía el bono, entonces ya no menos de 150 aunque la casa esté cayéndose, era 100 mil pesos lo que cobraban; cuando acá por ejemplo lo máximo que cobraran eran 30 mil, 50 mil pesos en una casa. Entonces la gente igual se aprovechó de esas circunstancias, sacó provecho, era obvio". (E2)

“... la casa, la casa, porque igual nosotros recién hacía cinco años que estábamos en nuestra casa po', como con proyectos de seguir arreglándola y todo eso, entonces llegar acá y pagar arriendos muy altos. ¿Y uno a veces le decía "pero por qué tan caro?", "bueno si a usted le están dando esa plata, entonces si no sale de su bolsillo qué problema se hace". (E3)

"Y los arriendos carísimos, nos sacaban los ojos". (E8)

“... porque igual acá vivir en Futaleufú no es la misma realidad que vivir en Puerto Montt, porque acá una, que acá es más caro vivir y en el invierno si tú no tienes plata para leña te entumes no más, se pasa frío; entonces había que organizarse, y otra cosa que los arriendos cuando pasó esto del volcán, los arriendos se fueron a las nubes, porque como la gente de Chaitén tenía plata para pagar arriendo, los arriendos se fueron carísimo y a toda la gente, por una casa mala o una casa buena, al final nos cobraban carísimo los arriendos”. (E7) 


\section{Categoría 3. Cambios en las condiciones de salud.}

\subsection{Aparición de enfermedades.}

Algunas de las enfermedades que empiezan a sufrir las personas surgen a partir de la evacuación y el desplazamiento, principalmente padecimientos asociados al estrés experimentado durante la situación de emergencia y, posteriormente, cuando deben reconstruir sus vidas en otros lugares.

“... entonces él se vio trabajando bajo mucha presión, tuvo mucho estrés y debido a eso él no se dio ni cuenta y entró en una ansiedad: comía, comía, comía, le subió el colesterol y tuvo un infarto... si... y claro después de eso, nosotros tuvimos que irnos a Puerto Montt porque él no podía recuperarse del infarto, si nosotros hace recién como un año que volvimos, que estuvimos en Puerto Montt”. (E3)

“... y después la operación, y después vuelta otra vez el tratamiento. Y eso me decía el doctor que todo el tema del estrés, la angustia que uno retuvo en el momento, no se dio cuenta lo que estaba pasando por su cuerpo, entonces afloraron esas enfermedades". (E4)

“...ya, empecé a sentirme mal, empecé a sentirme mal, me dolía el cuello, llegaba a mi casa y me empezaba a sentir mal físicamente, me daba dolor de cuello, me daba... musculares, dolores musculares, era atroz así como contracturas musculares. Empecé a sentir mucho mi cuerpo, muchas cosas así.. y empecé a tratar de cómo, yo creo que de una u otra forma mi cuerpo me estaba avisando que tenía que resolver los problemas, no esconderlos"... (E5).

\subsection{Dificultades en el estado de ánimo.}

En cuanto al estado de ánimo, las personas manifiestan que a partir de la evacuación, desplazamiento y cambios en sus condiciones de vida, experimentan sensaciones y sentimientos que antes no habían tenido mientras vivían en Chaitén. Es así como mencionan experimentar un malestar psicológico constante a partir de la ruptura de su línea de vida y de las experiencias que han tenido que enfrentar. 
“... empezamos a pasar el tiempo, a pasar el tiempo y me empezaba a sentir más mal y más mal; ya, me dijeron, ya vamos a ver, tú lo que tienes es una depresión, y a mí cuando me dijeron eso yo me molesté, yo me enojé y dije: cómo va a ser una depresión si me siento mal físicamente, yo no me quiero morir, no quiero suicidarme”. (E5)

“... y a mi lo que más me afectó cuando yo me vine acá en Futaleufú fue que cuando yo me vine acá, yo recién fue como que recién me di cuenta que mi papá no estaba, fue como que recién sentí la tristeza, el dolor y eso". (E8)

\section{Categoría 4. Desinformación}

\subsection{No saber lo que está sucediendo.}

Las personas evacuadas de Chaitén nunca contaron con información precisa. Lo que les daban era retazos de información que les impedía tener una idea clara de la situación, lo que a su vez dificultaba enormemente la comprensión cabal de la situación. La escasez de información, generadora de altos grados de incertidumbre, fue una constante desde el momento de la emergencia, durante la evacuación y después de esta. Obviamente la falta de información precisa llevó a que las personas tomaran decisiones basadas en intuiciones o rumores y careciendo del conocimiento de todo el contexto, de la situación en su totalidad, lo que en definitiva marca las decisiones que se tomaron por parte de las y los chaiteninos.

“... Pero era como un cierto temor por así decirlo, que tenía uno, porque uno veía los temblores, y como la información: unos decían una cosa, otros decían otra y uno escuchaba que la gente de la isla llamaban y decían otra cosa, y después las autoridades llamaban a la calma: "no, que no es así"; entonces era como que unos decían una cosa y las autoridades decían otra cosa, entonces uno no hallaba qué pensar. Sabía uno por lo que estaba sucediendo, como que entendíamos como que algo iba a pasar, pero no sabíamos realmente qué era, qué era”. (E3)

“... bueno, como decían en la radio: no, son placas tectónicas que se están moviendo, no hay problema, no hay nada. Escuchábamos nosotros los más abuelitos, los ancianitos que decían: no, ese no es ruido de las placas tectónicas, ese es ruido de volcán”. (E4) 
"Así que ya, los otros días dijimos: ¿qué será lo que pasa?, porque unos decían que podrían ser las placas tectónicas, otros que podía venir un terremoto fuerte, otros que tantas cosas... y las autoridades dijeron en ese tiempo, que no me acuerdo como se llamaba el intendente o alguien que estaba a cargo, fue a Chaitén y dijo que no había ningún tipo de peligro”. (E5)

"O sea yo creo que ninguno supo y yo creo que por eso tampoco perdimos, tampoco perdimos la confianza en volver, y esto fue porque nunca supimos que al caer eso que estaba sostenido, podríamos haber muerto todos. Yo creo que sí había gente, era muy poca la que sabía realmente lo que estaba pasando”. (E6)

\section{Análisis y conclusiones}

Los hallazgos encontrados en esta investigación nos indican que las experiencias de relocalización forzada de personas, gestionadas por instituciones y gobiernos (locales y nacionales) que centran casi completamente su atención en la recuperación de los aspectos infraestructurales y físicos, se convierten en generadoras de nuevas vulnerabilidades que ponen innecesariamente en riesgo el bienestar y la salud de las personas. El descuido de los factores psicosociales hace que, como en el caso de marras, surjan vulnerabilidades que nacen de cuatro condiciones o categorías, a saber, a) la ruptura del tejido social, b) los cambios en las condiciones de vida, c) los cambios en condiciones de salud y la d) desinformación. Hay que decir que desastres provocados por eventos naturales siempre dejan secuelas, aunque se haya hecho lo indecible por brindar apoyo integral a las personas (material y psicosocial). También hay que decir que ocuparse de restablecer las condiciones materiales (infraestructura, caminos, servicios) y de procurar refugio y cobijo a las personas que han perdido sus viviendas es un trabajo urgente, arduo y que merece reconocimiento. Sin embargo, también hay que indicar que el mal manejo por parte de las instituciones sociales y de gobierno, añade innecesariamente precariedad a las condiciones de vida de personas que, de la noche a la mañana, ven rotas sus cotidianidades y alteradas sus vidas de una forma dramática. En esta investigación encontramos que producto de intervenciones deficitarias por parte de las agencias encargadas, las personas se exponen a experiencias de desintegración familiar, a la pérdida de las redes de apoyo, a enfrentar un 
clima rechazo en las comunidades de "acogida", al extrañamiento que provoca el desarraigo y la pérdida del sentido de pertenencia, a la falta de empleo, a la incertidumbre con respecto al futuro y, como corolario, al padecimiento de enfermedades físicas y de trastornos psico-afectivos que responden a una experiencia que resulta traumática y que se vuelve más lesiva en la medida en que se descuidan aspectos medulares, relacionados con cuestiones que trascienden más allá de la recuperación de infraestructura y la dotación de viviendas o subsidios. Está comprobado que la existencia de una red de apoyo da un soporte excepcional que permite a las personas afrontar con mayor seguridad situaciones amenazantes (Karazec y Theorell, 1990; Grinberg y Grinberg, 1998) y en el caso de las personas de Chaitén queda absolutamente claro que sus familias representaban su soporte emocional más potente. Diseminar las familias no resulta entonces una medida que deba implementarse aun cuando las circunstancias hagan difícil la relocalización de toda la familia (nuclear y extensa) en un mismo sitio.

Prestar atención a las necesidades psicosociales de personas y poblaciones relocalizadas se convierte entonces en un imperativo de primer orden. Sin embargo, tal y como ha confirmado Beristain (2000) y ha ratificado esta misma investigación, se procrastina, generalmente de forma permanente, la atención de los aspectos psicosociales. Esto impide tomar consciencia de que tener en cuenta estos aspectos en todo momento permite:

“...entender los comportamientos y reacciones de la población; desarrollar planes de acción y prevención; comprender los mecanismos y acciones de apoyo mutuo de la gente en las situaciones de emergencia; y tener una comprensión global de la acción humanitaria, teniendo en cuenta las exigencias físicas, psicológicas y sociales de la población. (Beristain, 2000; p. 12)

La idea desde luego no es negar la relevancia que tiene garantizar tanto la seguridad fisica de las personas como reestablecer hasta donde sea posible las condiciones materiales que permitan atajar las amenazas que se ciernen sobre las personas. El punto a destacar es que es necesario ir más allá, esto es, comprender "cómo las personas viven, interpretan y reaccionan a estos acontecimientos. La gente tiene una experiencia previa, un pasado, una identidad que hay que comprender" (Beristain, 2000; p.16). 
En el caso de Chaitén, las autoridades de gobierno llamadas a atender la emergencia no siguieron las recomendaciones de Beristain. La falta de información precisa acerca de qué iba a pasar con la vida de las personas relocalizadas muestra hasta dónde puede llegar la falta de comprensión de la complejidad del fenómeno y hasta dónde importa priorizar el bienestar integral de las personas. La ambigüedad con respecto a si Chaitén iba a ser reconstruido o no (proceso que duró meses), si la gente podía o no retornar a su comunidad, es un claro ejemplo de la falta de sensibilidad y capacitación para manejar este tipo de situaciones. La falta de autonomía de las instancias que gestionaron el conflicto también llama poderosamente la atención, así como el manejo político y mediático de la crisis en medio de procesos electorales. Las personas requieren estar permanente informadas acerca de qué va pasar con ellos y con su comunidad; no permitir que la información fluya con transparencia y celeridad, pone en evidencia que la población es vista como una especie de objeto que hay que poner salvo en los términos concebidos por terceros, que no han analizado integralmente las vulnerabilidades de la población (las previas y las emergentes), y tampoco han identificado sus recursos y fortalezas para ser tomados en cuenta como agentes activos en el afrontamiento de la situación y, sobre todo, de su derecho de estar informados.

Si se pretende trabajar en comunidades o poblaciones afectadas por desastres, a partir de una conceptualización del riesgo y la vulnerabilidad entendidos como construcciones sociales, se hace necesario cambiar el enfoque de las intervenciones que se realizan. Esto implica trascender las acciones curativas para iniciar procesos de trabajo con las comunidades que permitan fortalecer sus capacidades "para transformar precisamente esas condiciones causales antes de que ocurra un desastre" (Wilches- Chaux, 1998, p.18). Es imperativo además trabajar con las vulnerabilidades de las poblaciones y no con sus necesidades, y considerar como un eje de trabajo fundamental "el fortalecimiento social y organizativo y el fortalecimiento en las actitudes y habilidades de las personas y las comunidades como elementos básicos que puedan romper la espiral de vulnerabilidad” (Pérez-Sales, 1998, p.8). Las intervenciones en desastres con enfoque psicosocial son fundamentales, ya que "los componentes psicosociales impregnan toda la vida de las personas y los grupos tras una catástrofe... si las personas pueden recuperar sus rutinas en poco tiempo, las consecuencias se atenúan” (Pérez-Sales, 1998, p.13). Incorporar la 
atención psicosocial de la emergencia y fomentar procesos participativos en la reconstrucción de la comunidad o en la definición de los nuevos destinos o en relación con la toma de decisiones acerca del futuro inmediato de las personas, reduciría o al menos no generaría nuevas vulnerabilidades en la población afectada por el desastre. Se hace necesario, pues, diseñar e implementar intervenciones comunitarias con estos objetivos, $\mathrm{y}$ sensibilizar a las autoridades y a los interventores con respecto de los beneficios que un trabajo con enfoque psicosocial brinda en estas situaciones.

Podrían relativizarse los alcances de los resultados de este estudio argumentando que se refieren muy puntualmente a una pequeña región de Chile localizada en la parte austral del continente. Sin embargo, consideramos que las vulnerabilidades emergentes identificadas en esta ocasión arrojan luz acerca de aspectos psicosociales a los que hay que prestar atención cuando se interviene en situaciones de desastre y especialmente de aquéllas que implican el desplazamiento forzado de personas.

\section{Referencias}

ACNUR. (1998). Principios Rectores de los Desplazamientos Internos. Recuperado de: https://www.icrc.org/spa/resources/documents/misc/5tdmhb.htm

Adamo, Susana. (2001). Emigración y ambiente: apuntes iniciales sobre un tema complejo. Papeles de Población, 29, 143-159.

Aguilera, Rodrigo. (2009) Chaitén: El lado B de la tragedia. Revista Nos Identifica. Recuperado en febrero de 2009 de: http://dl.dropboxusercontent.com/u/88541175/portadas/2009/02-concepcion/ index.html

Aigneren, Miguel (1999). Análisis de contenido: una introducción. Revista Electrónica La Sociología en sus escenarios, Número 3. Recuperado de: https://aprendeenlinea.udea.edu.co/revistas/index.php/ceo/article/view/1550/1207.

Álvarez-Gayou, Juan Luis. (2003). Cómo hacer investigación cualitativa. Fundamentos y metodología. México: Paidós. 
Anderson, Mary y Woodrow, Peter. (1989). Rising from the ashes: development strategies in time of disaster. Boulder, CO: Westview Press.

Arrieta, Carlos. (2017). El que se casa ¿quiere casa? Experiencia de relocalización en un proyecto de vivienda de interés social. Revista de Ciencias Sociales, 158, 125-147

Bauman, Zygmunt. (2011). Miedo líquido: la sociedad contemporánea y sus temores. Buenos Aires: Paidós.

Beristain, Carlos. (2000). Apoyo psicosocial en catástrofes colectivas. De la prevención a la reconstrucción. Venezuela: Melvin.

Berroeta, Héctor., Ramoneda, Alvaro., Rodríguez, Víctor, Di Masso, Andrés. y Vidal, Tomeu. (2015). Apego de lugar, identidad de lugar, sentido de comunidad y participación cívica en personas desplazadas de la comunidad de Chaitén. Magallania, 43 (3), 61-63.

Bordas, Dunot. (2000). Manual de investigación en Ciencias Sociales. México, D.F.: Editorial Limusa, S.A. de C.V. Grupo Noriega Editores.

Canales, Manuel. (2006). Metodología de Investigación Social. Santiago: LOM Ediciones.

Cohen, Roberta. (2009). El vacío institucional para los desplazados internos por desastres naturales. Revista Migraciones Forzadas, 32, 58 -59.

Contreras, Yenifer. (2008). Todo lo que sucedió el día de la evacuación masiva de Chaitén minuto a minuto. Recuperado de:

http://www.elvacanudo.cl/admin/render/noticia/14633

Chardon, Anne. (2010). Reasentar un hábitat vulnerable: teoría versus praxis. Revista INVI, 25, (70), $17-75$.

Duque, Juan. (2006). El reasentamiento poblacional: fenómeno social, político y de progreso. Estudios Socio Jurídicos, Bogotá Colombia 8, (1), 145-165.

Elias, Norbert y Stockton, John. (2000). Os estabelecidos e os outsiders. Brasil, Río de Janeiro: Jorge Zahar Ed.

Fernández, Ana María. (2005). Vulnerabilización de los jóvenes en Argentina: política y subjetividad. Revista Nómadas, 23, 132-139. Universidad Central, Colombia.

Fernández, Ana María. (2012). Jóvenes de vidas grises: Psicoanálisis y Biopolíticas. Buenos Aires: Nueva Visión.

García, Virginia. (2005). El riesgo como construcción social y la construcción social de riesgos. Revista Desacatos, 19, 11-24. 
García Canclini, Néstor. (2001). Culturas Híbridas. Estrategias para entrar y salir de la modernidad. Argentina: Editorial Paidós.

Grinberg, León y Grinberg, Rebeca. (1984). Psicoanálisis de la migración y del exilio. Madrid: Alianza Editorial.

Grinberg, León y Grinberg, Rebeca. (1998). Identidad y Cambio. España: Ediciones Paidós Ibérica.

Gonzáles, Teresa y Cano, Alejandra. (2010). Introducción al análisis de datos en investigación cualitativa: Tipos de análisis y proceso de codificación. Revista Nure Investigación, 45, 1 -10.

Jordana, Sebastian. (2009). Los habitantes de Chaitén están molestos por decisión de relocalizar el poblado. Recuperado de:

http://www.plataformaurbana.cl/archive/2009/01/30/los- habitantes-de-chaitenestan-molestos-por-decision-de-relocalizar-el-poblado/

Karasek, Robert y Theorell, Tores. (1990). Healthy work. Stress, productivity, and the reconstruction of working life. New York: Basic Books.

Labrín, José Miguel. (2010). Manual de Metodología Cualitativa. Marcos de referencia y nociones prácticas. Universidad de Chile, Instituto de Comunicación e Imagen.

Lavell, Allan. (2001) Sobre la Gestión del Riesgo: apuntes hacia una definición. Recuperado de: www.bvsde.paho.org/bvsacd/cd29/riesgo-apuntes.pdf

Organización de las Naciones Unidas. (2001). Menos Vulnerabilidad, Menos Desastres.

Recuperado de: https://www.unisdr.org/2001/campaign/pdf/kit_spanish.pdf.

Palacio, Jorge y Madariaga, Camilo. (2006). Lazos predominantes en las redes sociales personales de desplazados por violencia política. Investigación y Desarrollo, 14, (1), $86-119$.

Perevochtchikova, María y Lezama, José Luis. (2010). Causas de un desastre: Inundaciones del 2007 en Tabasco, México. Journal of Latin American Geography, 9, (2). Recuperado de: muse.jhu.edu/journal_of_latin_american_geography

Pérez-Sales, Pau. (1998). Intervención en catástrofes desde un enfoque psicosocial y comunitario. Átopos, 5, (18). Recuperado de http:// atopos.exlibrisediciones.com/pdf_01/intervención.pdf.

Porta, Luis y Silva, Miriam. (s.f.). La investigación cualitativa: El Análisis de Contenido en la investigación educativa. Recuperado de: 
https://www.uccor.edu.ar/paginas /REDUC/porta.pdf

Rojas, Jorge (2010). Vulnerabilidad social, neoliberalismo y desastre: sueños y temores de la comunidad desplazada/damnificada por el terremoto/tsunami. Sociedad Hoy, 19, 113-140.

Rosales, Vanessa y Salazar, Luis Fernando. (2010). Los procesos de reconstrucción: reto para la sociedad y para los gobiernos. Recuperado de: http://www.cne.go.cr/Documentos/mitigacion/el_caso_cinchona.pdf.

Sánchez, Alipio. (2007). Manual de Psicología Comunitaria: un enfoque integrado. España: Ediciones Pirámide.

Simmel, Georg. (2012). El extranjero. Sociología del extraño. Madrid: Ediciones Sequitur.

Schütz, Alfred. (2012). El forastero: ensayo de psicología social. En: G. Simmel, El extranjero: sociología del extraño. (pp. 27-42). Madrid: Ediciones Sequitur.

Tapia, Ricardo. (2012). Chaitén, Chile: Aprendizajes de un proceso de expulsión, reasentamiento y retorno humano en desarrollo como consecuencia de la erupción volcánica y aluvión del Volcán Chaitén en el año 2008.Centro de Investigación en Vulnerabilidades y Desastres Socio Naturales.

Uribe, Candy y Salina, Rolando. (2011). Procesos de precarización laboral y social, reflexiones en torno a sus consecuencias psicosociales. En A. Ovejero y J. Ramos (Eds). Psicología social crítica (pp. 264-271). Editorial Biblioteca Nueva, Madrid.

Wiesenfeld, Esther. (2000). Entre la prescripción y la acción: La brecha entre la teoría y la práctica en las investigaciones cualitativas. Forum: Qualitative Social Research, 1, (2). Recuperado de: http://www.qualitative-research.net

Wilches- Chaux, Gustavo. (1998). Auge, caída y levantada de Felipe Pinillo, mecánico y soldador o Yo voy a correr el riesgo. Guía de La RED para la gestión local del riesgo. Lima: La Red de Estudios Sociales en Prevención de Desastres en América Latina.

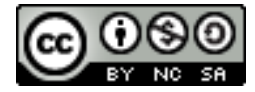

Esta obra está bajo una licencia de Creative Commons Reconocimiento-NoComercial-CompartirIgual 4.0 Internacional. 